\title{
An intelligent Fuzzy Inference System for Risk Estimation Using Matlab Platform: the Case of Forest Fires in Greece
}

\author{
Tsataltzinos $\mathbf{T}^{1}$, Iliadis $\mathbf{L}^{2}$, Spartalis $\mathbf{S}^{3}$ \\ ${ }^{1} \mathrm{PhD}$ candidate Democritus University of Thrace, Greece, tsataltzinos@yahoo.gr \\ ${ }^{2}$ Associate Professor Democritus University of Thrace, Greece, liliadis@fmenr.duth.gr \\ ${ }^{3}$ Professor Democritus University of Thrace, Greece, sspart@pme.duth.gr
}

\begin{abstract}
This paper aims in the design of an intelligent Fuzzy Inference System that evaluates risk due to natural disasters. Though its basic framework can be easily adjusted to perform in any type of natural hazard, it has been specifically designed to be applied in the case of forest fire risk in the area of the Greek terrain. Its purpose is to create a descending list of the areas under study, according to their degree of risk. This will provide important aid towards the task of distributing properly fire fighting resources. It is designed and implemented in Matlab's integrated Fuzzy Logic Toolbox. It estimates two basic kinds of risk indices, namely the man caused risk and the natural one. The fuzzy membership functions used in this project are the Triangular and the Semi-Triangular.
\end{abstract}

\section{Introduction}

Forest fire risk estimation is a major issue. The necessity for more efficient methods of fire fighting resources allocation becomes more and more urgent. This paper aims in the design of a new intelligent decision support system that performs ranking of the areas under consideration according to their forest fire risk. It is designed and implemented in Matlab and it uses fuzzy logic and fuzzy sets. The system assigns a degree of forest fire risk (DFFR) to each area by using Matlab's fuzzy toolbox and its integrated functions. The whole model that has been developed for this purpose consists of three distinct parts.

The first part is related to the determination of the main $\mathrm{n}$ risk factors (RF) affecting the specific risk problem. Three fuzzy sets (FS) were formed for each RF:

1. $\tilde{S}_{1}=\left\{\left(\mu_{\mathrm{j}}\left(\mathrm{A}_{\mathrm{j}}\right), \mathrm{X}_{\mathrm{i}}\right)\right.$ (forest departments $\mathrm{A}_{\mathrm{j}}$ of small risk) $\left./ \mathrm{j}=1 \ldots \mathrm{N}, \mathrm{i}=1 \ldots \mathrm{M}\right\}$
2. $\tilde{S}_{2}=\left\{\left(\kappa_{\mathrm{j}}\left(\mathrm{A}_{\mathrm{j}}\right), \mathrm{X}_{\mathrm{i}}\right)\right.$ (forest departments $\mathrm{A}_{\mathrm{j}}$ of average risk) $\left./ \mathrm{j}=1 \ldots \mathrm{N}, \mathrm{i}=1 \ldots \mathrm{M}\right\}$

Please use the following format when citing this chapter:

Tsataltzinos, T., Iliadis, L. and Spartalis, S., 2009, in IFIP International Federation for Information Processing, Volume 296; Artificial Intelligence Applications and Innovations III; Eds. Iliadis, L., Vlahavas, I., Bramer, M.; (Boston: Springer), pp. 303-310. 
3. $\tilde{S}_{3}=\left\{\left(\lambda_{\mathrm{j}}\left(\mathrm{A}_{\mathrm{j}}\right), \mathrm{X}_{\mathrm{i}}\right)\right.$ (forest departments $\mathrm{A}_{\mathrm{j}}$ of high risk) $\left./ \mathrm{j}=1 \ldots \mathrm{N}, \mathrm{i}=1 \ldots \mathrm{M}\right\}$

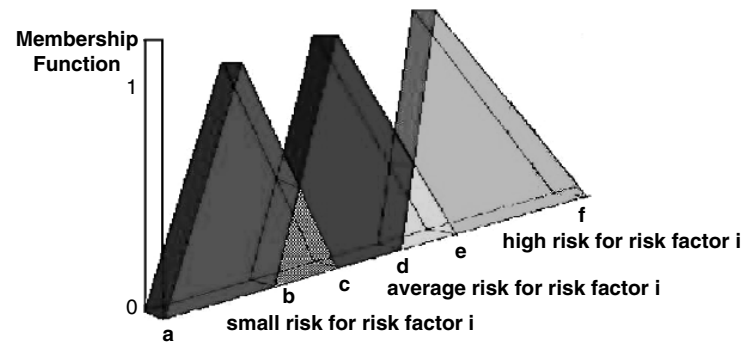

Fig. 1. Degree of fuzzy risk of forest department $\mathrm{j}$ for risk factor $\mathrm{i}$

The risk factors are distinguished in two basic categories; Human factors and Natural ones (Kailidis, 1990). Each one of these general risk types consists of several sub-factors that influence in their own way the final risk degree (RD).

The second part was the design of the system's main rule set that would perform the unification of the partial degrees of risk and the output of the unified risk index (URI). These rules are distinct for each risk factor and most of them are specified in bibliography (Kailidis, 1990). The greater the number of factors is, the greater the number of rules required. This is the typical problem of combinatorial explosion in the development of rule based knowledge systems.

To avoid the use of a huge number of rules, so that the project retains its simplicity, the factors were divided into smaller subgroups according to their nature. Decision tables were created and used for each subgroup. In this way the number of rules was minimized significantly.

The third part of the development process was the application of the rule set for the production of the URI. The URI can be produced by applying various types of fuzzy relations to perform fuzzy AND, fuzzy OR operations between the fuzzy sets (and consequently between partial risk indices). The functions for the conjunction are called T-norms and for the union T-conorms or S-norms (Kandel A., 1992).

\section{Basic Design Principles of the Intelligent System}

The System was developed using Matlab's integrated Fuzzy Logic Toolbox. The row data was input into an MS Access database and extracted into MS Excel datasheets. Next, each column of the data was extracted into a separate Excel file to form an input variable for Matlab. Using the xlsread and xlswrite commands of the fuzzy toolbox, the final results were also extracted into an Excel file. The triangular fuzzy membership function was implemented by the triamf function of the fuzzy toolbox. This Project applied the Matlab's integrated Mamdani Inference 
method, which operates in a forward chaining mode. The Mamdani inference system comprises of five parts:

1. Fuzzyfication of input with the Triangular membership function (Function 1)

2. Application of fuzzy operators. OR operation is performed by $\mu(x)=\max \left(x_{n}\right)$, while AND operation by using the algebraic product $\mu(x)=x_{1} x_{2} \quad x_{n}$

3. Application of the implication method $(\min ) \mu(x)=\min \left(x_{n}\right)$

4. Aggregation of output values with the use of $\max$ function $\mu(x)=\max \left(x_{n}\right)$

5. Defuzzification on the output with the centroid method $\mu(\chi)=\frac{\int_{\chi} x f(x) d x}{\int_{\chi} f(x) d x}$

\section{Materials and Methods}

\subsection{Determination of the risk factors}

The problem of forest fire risk estimation can be faced as a daily measured process or as an index indicating the risk of having high volume of forest incidents on an annual basis. Both of these orientations are influenced by a great number of parameters (Kailidis 1990). This project aims in the estimation of the annual forest fire risk due to the lack of daily measurements. To do this, two basic data groups were gathered. Each one of them is consisted of the following factors that can be seen in Table 1.

Table 1. Factor Groups

\begin{tabular}{ll}
\hline Human Factors & Natural Factors \\
\hline Population Density & Average Annual Temperature \\
Tourism & Average Annual Humidity \\
Land Value & Average Altitude \\
Other - User' s estimation & Percentage of Forest Cover \\
& Average Wind speed \\
\hline
\end{tabular}

In the case of the human risk factors, the population density and the tourism data was gathered from the General Secretariat of National Statistical Service of Greece. The land value was estimated with the use of the previous two. The bigger the population density of a forest department is and the greater its tourist development the higher its land value. The value is represented in pure numbers from 1 
to 10 . The fourth factor is input to exploit the experience and the intuition of a forest fire expert on the risk degree of an area.

In the case of natural factors, the Average Annual Temperature, Humidity and Wind Speed were used. For better results, the above three factors' data were separated into seasons or months, because the risk has a seasonal nature. Yet the system is capable of using even daily updates of these data to produce risk analysis on a more frequent basis. The percentage of forest cover does not include the kind of vegetation of each forest department due to the fact that this is a pilot effort. The system also uses the Average Altitude of every data point as a risk factor.

The DSS was applied in all of the Greek territory. Meteorological and morphological data was gathered from Greek public services. Population density data was gathered from General Secretariat of National Statistical Service of Greece. The forest fire data used cover the period between 1983 and 1994, and the population census of 1991. The results were extracted into different MS Excel files. This is a pilot application just to indicate the performance validity of the prototype.

\subsection{The fuzzy rule system}

Fuzzy Logic (FL) and Fuzzy Sets (FS) can provide aid towards modeling the human knowledge and real world concepts (Leondes, 1998). For example the modeling of the concept "Hot area" in terms of average temperature, is both subjective and imprecise so it can be considered as a fuzzy set (FS). It is clear that real world situations can be described with the use of proper linguistics, each one defined by a corresponding FS. For every FS there exists a degree of membership (DOM) $\mu_{\mathrm{s}}(\mathrm{X})$ that is mapped on $[0,1]$. For example every forest department belongs to the FS "fire risky forest department" with a different degree of membership (Kandel, 1992). The functions used to define the DOM are called fuzzy membership functions (FMF) and in this project the triangular FMF (TRIAMF) and the semitriangular FMF (semi-TRIAMF) were applied (Iliadis L. 2005). Functions 1 and 2 below represent the TRIAMF and semi-TRIAMF

Function 1

$$
\mu_{s}(X)=\left\{\begin{array}{l}
0 \text { if } X<a \\
(X-a) /(c-a) \text { if } X \in[a, c] \\
(b-X) /(b-c) \text { if } X \in[c, b] \\
0 \text { if } X>b
\end{array}\right.
$$

Function 2

$$
\mu_{s}(X)=\left\{\begin{array}{l}
0 \text { if } X<a \\
(X-a) /(b-a) \text { if } X \in[a, b]
\end{array}\right.
$$

Singleton functions were used to determine the boundaries of the membership functions. The system assigns each forest department three Partial Risk Indices (PRI), for every one of the nine factors that are taken under consideration, as it is shown below: 
1. Low Danger due to each factor

2. Medium Danger due to each factor

3. High Danger due to each factor

Table 2. Values of the Singleton Fuzzy membership functions min and max

\begin{tabular}{lll}
\hline Min & Factor & Max \\
\hline 0 & Average Temperature & 38 \\
0 & Average Humidity & 80 \\
1 & Average Wind speed & 3 \\
0 & Average Height & 1280 \\
0 & Average Forest Cover & 2 \\
0 & Population Density & 300000 \\
0 & Tourism & 10 \\
0 & Land Value & 10 \\
0 & Other - Experts opinion* & 10 \\
$*$ depends on the expert. The min and max values are not \\
necessarily those that are shown on this table
\end{tabular}

For each factor the minimum and maximum boundaries of its fuzzy membership function are shown in table 2 above. This method allows the use of any kind of data and does not need specific metrics for every factor. Due to this fact, there was no need to do any changes in the row data provided by the Greek national services. The above steps resulted in having 27 different PRIs. The more detailed the linguistics become the greater the number of PRIs. Those 27 PRIs are too many and not quite helpful. The next step was to unify them in one Unified Risk Index (URI). To do this, this project had to take into consideration the human experience and to apply the rules that a human expert would use. For example if it is known that an area has great population and tourism (which results in great land value), it is near the sea (which means low altitude) and it has great forest cover, then it definitely is a very dangerous area and needs to have our attention. In this example, four parameters were used.

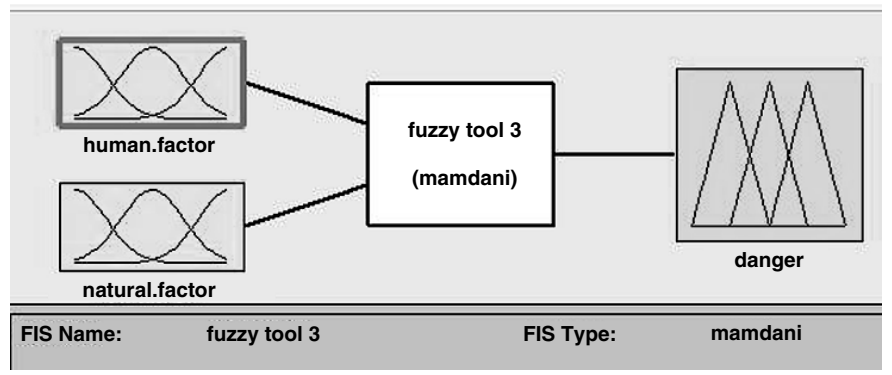

Fig. 2. Fuzzy toolbox sample 
If two FSs are used for each factor then the proper number of rules that should be used is $2^{4}=16$. In this project the number of rules that had to be applied was $3^{9}=19683$. To make the number of rules smaller, the factors had to be combined into small subgroups of two or three, as shown in figure 2. This resulted in a much smaller number of rules, much easier to understand and apply. The total amount of rules required was $3^{3}+6 * 3^{2}=81$. For example, to combine the Population Density (Pop) and the Tourism (Tour) factors in one subgroup named "Measurable Human Factors" (MHF) the following 9 rules had to be applied:

1. If Pop is low and Tour is low then MHF is low

2. If Pop is average and Tour is average then MHF is average

3. If Pop is high and Tour is high then MHF is high

4. If Pop is low and Tour is average then MHF is average

5. If Pop is low and Tour is high then MHF is high

6. If Pop is average and Tour is low then MHF is average

7. If Pop is average and Tour is high then MHF is high

8. If Pop is high and Tour is average then MHF is high

9. If Pop is high and Tour is low then MHF is high

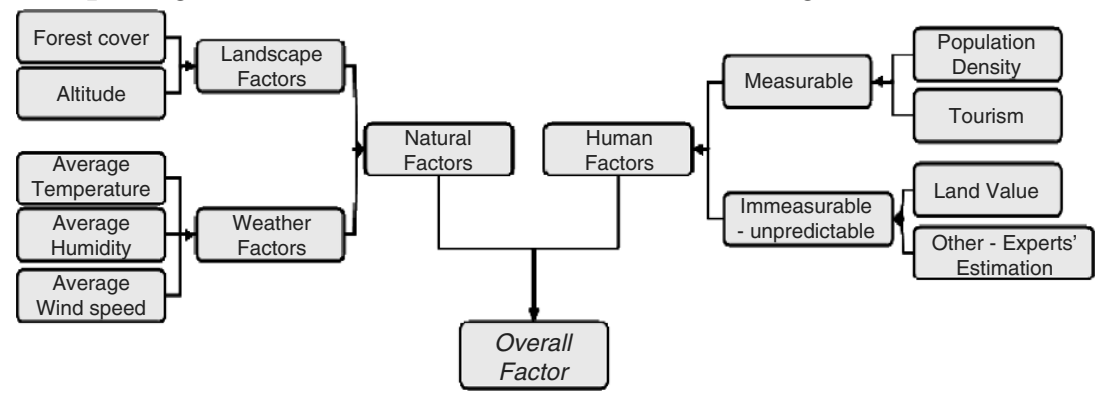

Fig. 3. Categorization of the risk factors

Following the logic of the above structure (Fig. 3) the number of rules was reduced significantly making the operation of the system much simpler. Combined with the proper decision tables (Table 3 ) the total number of rules was reduced to 73.

\section{$5 \quad$ Results and Discussion}

This model and its corresponding intelligent information system provide a descending ranking of the forest departments in Greece according to their forest fire risk. The final membership values of all forest departments can only be compared between each other. The bigger the difference between the final values of two departments is, the bigger the difference in actual risk they have. To check the results validity, each year's data was processed separately. The resulting descending 
Table 3. Decision Table sample

\begin{tabular}{|c|c|c|c|c|c|c|c|c|c|c|c|c|c|c|c|c|c|c|c|c|c|c|c|c|c|c|c|}
\hline Temperature & $\mathrm{L}$ & $\mathrm{L}$ & $\mathrm{L}$ & $\mathrm{L}$ & $\mathrm{L}$ & $\mathrm{L}$ & $\mathrm{L}$ & $\mathrm{L}$ & $\mathrm{L}$ & $\mathrm{M}$ & $\mathrm{M}$ & $\mathrm{M}$ & $\mathrm{M}$ & $\mathrm{M}$ & $\mathrm{M}$ & $\mathrm{M}$ & $\mathrm{M}$ & $\mathrm{M}$ & $\mathrm{H}$ & $\mathrm{H}$ & $\mathrm{H}$ & $\mathrm{H}$ & $\mathrm{H}$ & $\mathrm{H}$ & $\mathrm{H}$ & $\mathrm{H}$ & $\mathrm{H}$ \\
\hline Wind & $\mathrm{L}$ & $\mathrm{L}$ & $\mathrm{L}$ & $\mathrm{M}$ & $\mathrm{M}$ & $\mathrm{M}$ & $\mathrm{H}$ & $\mathrm{H}$ & $\mathrm{H}$ & $\mathrm{L}$ & $\mathrm{L}$ & $\mathrm{L}$ & $\mathrm{M}$ & $\mathrm{M}$ & $\mathrm{M}$ & $\mathrm{H}$ & $\mathrm{H}$ & $\mathrm{H}$ & L & $\mathrm{L}$ & $\mathrm{L}$ & $\mathrm{M}$ & $\mathrm{M}$ & $\mathrm{M}$ & $\mathrm{H}$ & $\mathrm{H}$ & $\mathrm{H}$ \\
\hline \begin{tabular}{|l|} 
Humidity \\
\end{tabular} & $\mathrm{L}$ & $\mathrm{M}$ & \begin{tabular}{|l|}
$\mathrm{H}$ \\
\end{tabular} & $\mathrm{L}$ & $\mathrm{M}$ & $\mathrm{H}$ & \begin{tabular}{|l|l}
$\mathrm{L}$ \\
\end{tabular} & $\mathrm{M}$ & $\mathrm{H}$ & $\mathrm{L}$ & $\mathrm{M}$ & $\mathrm{H}$ & $\mathrm{L}$ & $\mathrm{M}$ & $\mathrm{H}$ & $\mathrm{L}$ & $\mathrm{M}$ & $\mathrm{H}$ & $\mathrm{L}$ & $\mathrm{M}$ & $\mathrm{H}$ & $\mathrm{L}$ & $\mathrm{M}$ & $\mathrm{H}$ & \begin{tabular}{|l|l}
$\mathrm{L}$ \\
\end{tabular} & $\mathrm{M}$ & $\mathrm{H}$ \\
\hline Low Danger & $\mathrm{X}$ & $\mathrm{X}$ & $\mathrm{X}$ & & $\mathrm{X}$ & $\mathrm{X}$ & & $\mathrm{X}$ & $\mathrm{X}$ & & & & & & & & & & & & & & & & & & \\
\hline Medium Danger & & & & $\mathrm{x}$ & & & $\mathrm{x}$ & & & $\mathrm{X}$ & $\mathrm{X}$ & $\mathrm{X}$ & & $\mathrm{X}$ & $\mathrm{X}$ & & & $\mathrm{X}$ & & $\mathrm{x}$ & $\mathrm{x}$ & & & & & & \\
\hline High Danger & & & & & & & & & & & & & $\mathrm{X}$ & & & $\mathrm{X}$ & $\mathrm{X}$ & & $\mathrm{x}$ & & & $\mathrm{X}$ & $\mathrm{X}$ & $\mathrm{X}$ & $\mathrm{X}$ & $\mathrm{X}$ & $\mathrm{X}$ \\
\hline
\end{tabular}

\begin{tabular}{|c|c|c|c|c|c|c|c|c|c|c|c|c|c|c|c|c|c|c|c|}
\hline Final Table & \multicolumn{7}{|c|}{ Group 1} & \multicolumn{7}{|c|}{ Group 2} & \multicolumn{5}{|c|}{ Group 3} \\
\hline Temperature & $\mathrm{L}$ & $\mathrm{L}$ & $\mathrm{L}$ & $\mathrm{L}$ & $\mathrm{L}$ & $\mathrm{L}$ & L & $\mathrm{M}$ & $\mathrm{M}$ & $\mathrm{M}$ & $\mathrm{M}$ & $\mathrm{M}$ & $\mathrm{M}$ & $\mathrm{M}$ & $\mathrm{H}$ & $\mathrm{H}$ & $\mathrm{H}$ & $\mathrm{H}$ & $\mathrm{H}$ \\
\hline Wind & $\mathrm{L}$ & $\bar{M}$ & $\mathrm{M}$ & $\mathrm{M}$ & $\mathrm{H}$ & $\mathrm{H}$ & $\mathrm{H}$ & $\mathrm{L}$ & $\mathrm{M}$ & $\mathrm{M}$ & $\mathrm{M}$ & $\mathrm{H}$ & $\mathrm{H}$ & $\mathrm{H}$ & $\mathrm{L}$ & $\mathrm{L}$ & $\mathrm{L}$ & $\mathrm{M}$ & $\mathrm{H}$ \\
\hline Humidity & - & $\mathrm{L}$ & $\mathrm{M}$ & $\mathrm{H}$ & $\mathrm{L}$ & M & $\mathrm{H}$ & - & $\mathrm{L}$ & $\mathrm{M}$ & $\mathrm{H}$ & $\mathrm{L}$ & $\mathrm{M}$ & $\mathrm{H}$ & $\mathrm{L}$ & $\mathrm{M}$ & $\mathrm{H}$ & & \\
\hline Low Danger & $\mathrm{x}$ & & $\mathrm{X}$ & $\mathrm{X}$ & & $\mathrm{X}$ & $\bar{x}$ & & & & & & & & & & & & \\
\hline Medium Danger & & $\mathrm{X}$ & & & $\mathrm{X}$ & & & $\mathrm{X}$ & & $\mathrm{X}$ & $\mathrm{X}$ & & & $\mathrm{X}$ & & $\mathrm{X}$ & $\mathrm{X}$ & & \\
\hline High Danger & & & & & & & & & $X$ & & & $\mathrm{X}$ & $\mathrm{X}$ & & $\mathrm{X}$ & & & $\mathrm{x}$ & $\mathrm{X}$ \\
\hline
\end{tabular}

\begin{tabular}{|l|}
\hline \multicolumn{1}{|c|}{ Legend } \\
\hline $\mathrm{L}=$ Low \\
\hline $\mathrm{M}=$ Medium \\
\hline $\mathrm{H}=$ High \\
\hline
\end{tabular}

list produced from every year's data was compared to the list obtained from the ranking of the departments on their actual annual number of forest fires of the following year. The compatibility of this method to the actual annual forest fire situation varied from $52 \%$ to $70 \%$ (Table 4). In some cases, forest departments used to mark as "forest fire" agricultural fires (caused on purpose) which makes the actual logic of the Ruleset less efficient. However in a future effort this type of data should be diminished from the fire database.

Table 4. Results.

$\begin{array}{lllllllllll}83-84 & 84-85 & 85-86 & 86-87 & 87-88 & 88-89 & 89-90 & 90-91 & 91-92 & 92-93\end{array}$

Compatibility with the

following year's actual

ranking for the risky

$\begin{array}{llllllllll}52 \% & 58 \% & 62 \% & 62 \% & 66 \% & 64 \% & 62 \% & 70 \% & 54 \% & 58 \%\end{array}$ area fuzzy set

The final ranking of all the forest departments remains almost the same despite the use of other fuzzy membership functions. The system was also tested with the use of Trapezoidal, semi-Trapezoidal and Sigmoid membership functions and the differentiation in the results was not significant. Nevertheless, even if 52\% to $70 \%$ may not seem an impressively reliable performance from the statistical point of view, it is actually a performance offering a very good practical application. Obviously there would be many governments that would be vary happy if they could know from the previous year $52 \%-70 \%$ of the areas that are threatened seriously by forest fires.

Testing showed that the years that had luck of detailed data for many forest departments resulted in low compatibility, while on the other hand the results were pretty impressive when there was enough data for all the departments. This also was a first attempt to use detailed data for the human factors. The first tests included only the "population density" and "tourism" factors. These tests resulted in a maximum compatibly of $70 \%$. 
The more detailed the data becomes on the human factors and with the help of a proper human expert the better the accuracy of the system would become. Forest fires can occur due to a great number of factors. Many of those factors are extremely unpredictable and immeasurable. These facts make the fire estimation a complicated problem that can be studied with the use of fuzzy logic. This system uses an alternative way of thinking and offers a different approach. The fact that it can use any kind of data available and that it can produce results as soon as the data is inserted, makes it a valuable tool for estimating which forest department is in danger. On the other hand, due to the fact that human behavior is pretty unpredictable, the expert's opinion is necessary to enable the production of better results or even to perform various scenarios.

The system has shown that it is quite useful and that it can improve its performance if more data is gathered. It will also be expanded towards the estimation of the daily forest fire risk which can be seen as the problem of having favorable forest fire ignition and acceleration conditions.

\section{References}

1. Tsataltzinos T. (2007) "A fuzzy decision support system evaluating qualitative attributes towards forest fire risk estimation", Proceedings 10th International Conference on Engineering Applications of Neural Networks, Thessaloniki, Hellas, August 2007.

2. Iliadis L. (2005) "A decision support system applying an integrated Fuzzy model for long - term forest fire risk estimation" Environmental Modelling and Software, Elsevier Science, Vol.20, No.5, pp.613-621, May 2005.

3. Iliadis L., Maris F., Tsataltzinos T. (2005). "An innovative Decision Support System using Fuzzy Reasoning for the Estimation of Mountainous Watersheds Torrential Risk: The case of Lakes Koroneia and Vovli", Proceedings IUFRO Conference "Sustainable Forestry in theory and practice: recent advances in inventory and monitoring statistics and modeling information and knowledge management and policy science" Pacific Northwest Research Station GTR-PNW-688, University of Edimburgh, UK,

4. Kandel A., 1992, Fuzzy Expert Systems. CRC Press. USA.

5. Kecman V., 2001, Learning and Soft Computing. MIT Press. London England.

6. Leondes C.T., 1998, "Fuzzy Logic and Expert Systems Applications", Academic Press. California USA.

7. Iliadis L., Spartalis S., Maris F., Marinos D. 2004 "A Decision Support System Unifying Trapezoidal Function Membership Values using T-Norms". Proceedings International Conference in Numerical Analysis and Applied Mathematics (ICNAAM), J. Wiley-VCH Verlag GmbH Publishing co., Weinheim Germany.

8. Zhang J.X., Huang C.F., 2005. "Cartographic Representation of the Uncertainty related to natural disaster risk: overview and state of the art", LNAI Vol. 3327, pp. 213-220

9. Nguyen H., Walker E., 2000. "A First Course in Fuzzy Logic", Chapman and Hall, Library of the Congress, USA

10. Cox E., 2005. Fuzzy Modeling and Genetic Algorithms for Data Mining and Exploration, Elsevier Science, USA

11. E A Johnson, Kiyoko Miyanishi, 2001, "Forest Fire: Behavior and Ecological Effects"

12. J Kahlert, H Frank, 1994, "Fuzzy-Logik und Fuzzy-Control"

13. Mamdani, E.H. and S. Assilian, "An experiment in linguistic synthesis with a fuzzy logic controller," International Journal of Man-Machine Studies, Vol. 7, No. 1, pp. 1-13, 1975.

14. Kailidis D. 1990, "Forest Fires" 